\title{
Light emitting fabrics for PDT: technology and results of clinical studies.
}

Serge R. Mordon ${ }^{1} *$, Elise Thecua ${ }^{1}$, Fabienne Lecomte ${ }^{1}$, Anne Sophie. Vignion-Dewalle ${ }^{1}$, Pascal Deleporte $^{1}$, Cyril Maire ${ }^{1,2}$ Henry Abi-Rached ${ }^{1,2}$, Claire Vicentini ${ }^{1,2}$, Theresa Hommel ${ }^{3}$, R. Markus Szeimies ${ }^{3}$, Laurent Mortier ${ }^{1,2}$

\footnotetext{
${ }^{1}$ Univ. Lille, INSERM, CHU Lille, U1189 - ONCO-THAI - Image Assisted Laser Therapy for Oncology, F59000 Lille, France

${ }^{2}$ Department of Dermatology, CHU Lille, F-59000 Lille, France

${ }^{3}$ Department of Dermatology and Allergology, Klinikum Vest GmbH, D-45657 Recklinghausen, Germany
}

\begin{abstract}
A homogeneous and reproducible fluence rate delivery during clinical PDT plays a determinant role in preventing under- or overtreatment. In Dermatology, topical PDT has been carried out with a wide variety of light sources delivering a broad range of light doses. However, these light sources do not deliver a uniform light distribution on the skin due to their structure and morphology and the complexities of the human anatomy. The development of a flexible light source able to generate uniform light on all its surface would considerably improve the homogeneity of light delivery.
\end{abstract}

The integration of plastic optical fibers (POF) into textile structures offers an interesting alternative.

The homogeneous light side-emission from the fabric is obtained by controlling the bending angles of POF inside the LEF due to specific architecture generated by knitting of textile structure. LEF of different surfaces can be easily manufactured (up to $500 \mathrm{~cm}^{2}$ The LEF thickness is less than $1 \mathrm{~mm}$. The mean irradiance is typically $2.5 \mathrm{~mW} . \mathrm{cm}^{2}{ }^{2}$. W- 1 with heterogeneity of $12.5 \%$ at any point of the LEF. The temperature elevation remains below $1^{\circ} \mathrm{C}$. These LEF were evaluated in Dermatology for the treatment of Actinic Keratosis. Two clinical evaluation were performed. The first one was a monocentric, randomized, controlled, phase II clinical study (ClinicalTrials.gov Identifier: NCT03076918). Twenty five (25) patients with grade I-II actinic keratosis (AK) of the forehead and scalp were treated with methyl aminolevulinate photodynamic therapy in two symmetrical areas. One area was treated with the conventional LED panel (154 AK), whereas the other area was treated with the LEF device (156 AK). The second clinical was performed in 2 centers. This new LEF device was a more ergonomic and compact version of the original system developed for FLEXI-PDT. In this clinical study (ClinicalTrials.gov Identifier: NCT03076892), the irradiance has been reduced from 12.3 $\mathrm{mW} / \mathrm{cm}^{2}$ to $1.3 \mathrm{~mW} / \mathrm{cm}^{2}$ and the light dose from $37 \mathrm{~J} / \mathrm{cm}^{2}$ to $12 \mathrm{~J} / \mathrm{cm}^{2}$. Compared to Conventional PDT, the 2 protocols clearly shown that LEF are equivalent and even superior in terms of efficacy for treating AK of the forehead and scalp. However, the use of LEF resulted in much lower pain scores and fewer adverse effects. In conclusion, thanks to LEF, PDT of AK 
can be conducted in all weather conditions, in any geographic location, year-round and benefits from the optimal adaptability of the flexible, light-emitting, fabrics to the treatment area. At last, LEF can be easily can be easily manufactured in large series.

Keywords: Photodynamic Therapy, Light Emitting Fabric, Textile, Laser, Actinic Keratosis, Clinical Evaluation

* serge.mordon@inserm.fr / phone: +33 (0) 320446708 : www.oncothai.fr

\section{INTRODUCTION}

\section{PDT in Dermatology}

Photodynamic therapy (PDT) is considered to be a promising method for treating various types of cancer. A photosensitizer (or a precursor) is used to make the cells sensitive to light. Eventually, the tumor cells are exposed to the light leading the photosensitizer to interact with the oxygen in the cell. This photophysical mechanism produces toxic substances that destroy the tumor cell. Afterwards, the cell dies off and is replaced by healthy tissue. A homogeneous and reproducible fluence delivery rate during clinical photodynamic therapy (PDT) plays a determinant role in preventing under- or overtreatment. In dermatology, PDT is used to treat actinic keratoses. AKs are common pre-invasive cancerous lesions in sunexposed skin which negatively affect the quality of life in patients and may progress to invasive squamous cell carcinoma (SCC). AK usually develop on areas that are frequently exposed to the sun (e.g., face, ears, scalp, neck, forearms, and back of the hands). Studies have shown that if AK are untreated, AK may regress, or alternatively, may progress to SCC, with significant morbidity and possible lethal outcome. Predicting which AKs may progress to SCC is not possible, nor is the conversion rate for an AK to SCC clear: the transformation rate from an AK lesion to SCC within one year has been reported to be $<1: 1000$. The malignant potential and the fact that it is impossible to predict which AK will evolve into SCC, have led to the common consensus that AKs have to be treated. Because of the high prevalence of AKs, their treatment represents a substantial workload, and must therefore be efficacious and easy to perform. Moreover, for patients an ideal treatment should be well tolerated and result in good cosmesis. The most commonly used treatments for AK are cryotherapy, topical chemotherapy and, more recently, photodynamic therapy (PDT) [1] However, for this application, PDT is carried out with a wide variety of light sources delivering a broad range of more or less adapted light doses. Due to the complexities of the human anatomy, these light sources do not in fact deliver a uniform light distribution to the skin. For example, in the case of the LED system used usually in Dermatology, Moseley et al demonstrated that the irradiance may be as low as $38 \%$ of the central area at a distance of only $2 \mathrm{~cm}$ [2]. 
Therefore, the development of flexible light sources would considerably improve the homogeneity of light delivery. The integration of optical fiber into flexible structures could offer an interesting alternative [3].

The paper aims to describe briefly the technology used to develop Light Emitting Fabrics (LEF) and their use in Dermatology to treat Actinic Keratosis by Photodynamic Therapy. In fact, two clinical protocols were designed to perform the evaluation of this new and innovative illumination device. The first one is called FLEXI-PDT, the second one is the Phosistos protocol (P-PDT). The second one was carried out with an improved version of the original system developed for FLEXI-PDT. In P-PDT, the irradiance has been reduced from $12.3 \mathrm{~mW} / \mathrm{cm}^{2}$ to 1.3 $\mathrm{mW} / \mathrm{cm}^{2}$ and the light dose from $37 \mathrm{~J} / \mathrm{cm}^{2}$ to $12 \mathrm{~J} / \mathrm{cm}^{2}$. Furthermore, the device has been redesigned so as to be more ergonomic and compact.

\section{MATERIALS \& METHODS}

\section{Light Emitting Fabrics}

Different technologies exist for the manufacturing of LEF [3]. Schematically, the technology developed for this clinical evaluation consists in the integration of step index optical fibers (TORAY, Tokyo, JAPAN) with a polymethyl methacrylate (PMMA) core and a fluorinated cladding within a fabric structure during a knitting process. Controlling macrobendings and yarn tension during the knitting process is the key factor for a homogeneous light emission over the entire surface of the sample. Produced in one step by warp knitting technology, LEF are made of polyester yarn and embed $37 \mathrm{POF} / \mathrm{cm}^{2}$ and can have an effective area over 500 $\mathrm{cm}^{2}$ while keeping flexible and conformable properties $[4,5]$

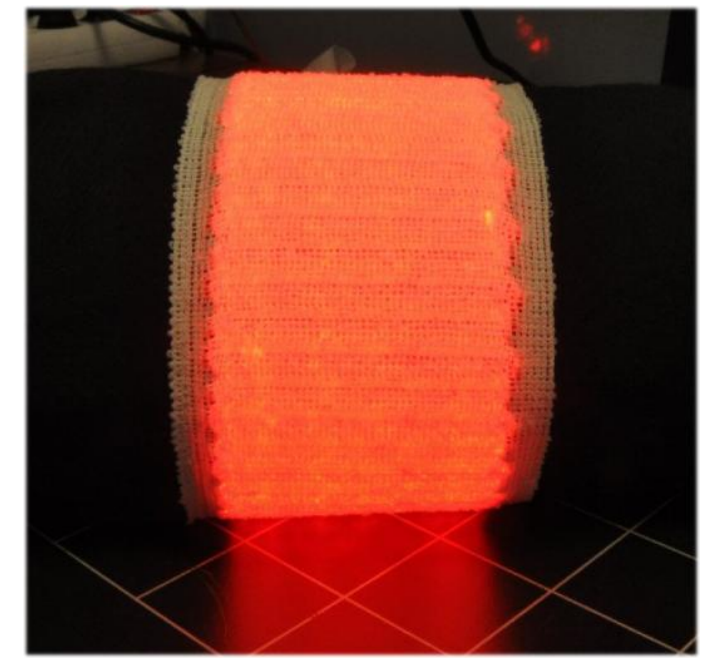

Figure 1: Light Emitting Fabrics obtained by a knitting process connected to a red laser source $(635 \mathrm{~nm})$ 


\section{Flexitheralight}

As illustrated in Figure 2, this device consists of a power and control unit including three laser diodes, each connected to a flexible light-emitting fabric sample of size $21.5 \mathrm{~cm} \times 5 \mathrm{~cm}$, which is made of biocompatible optical fibres (18). The fabric samples, which were sewn together (for a total area of $3 \times 21.5 \mathrm{~cm} \times 5 \mathrm{~cm}=322.5 \mathrm{~cm}^{2}$ ), sequentially emit $635 \mathrm{~nm}$ red light at low fluence rate $\left(12.3 \mathrm{~mW} / \mathrm{cm}^{2}\right)$ for one minute, such as a fractionated irradiation (1 minute light, 2 minutes dark) is achieved. An irradiation time of two and a half hours enables to deliver a total light dose of about $37 \mathrm{~J} / \mathrm{cm}^{2}$ anywhere in the treated area $\left(12.3 \mathrm{~mW} / \mathrm{cm}^{2} \times 9000 \mathrm{~s} \times 1\right.$ minute light / (1 minute light +2 minutes dark)).

FLEXI-PDT has two main features. On the one hand, the short incubation time should allow a continuous activation of small amounts of porphyrins during its formation and a reduction of patient-reported pain as is the case for protocols involving irradiation with daylight. On the other hand, the fabrics are flexible and allow an optimal conformation to the area to be treated, thus leading to a more homogeneous irradiation than that delivered by the standard rigid light sources.

The FLEXITHERALIGHT protocol, developed within the FLEXITERALIGHT project supported by the French National Research Agency (ANR) (Projet-ANR-12-EMMA-0018) (http://www.flexitheralight.com/), involves a 30-minute incubation with MAL followed by a $2.5 \mathrm{~h}$ irradiation with a light-emitting fabric-based device. Due to the short incubation time, the FLEXITHERALIGHT protocol (FLEXI-PDT) should provide a nearly pain-free, all year round alternative to C-PDT. Moreover, the high flexibility of the light-emitting fabric-based device ensures an optimal conformation of the device to the area to be treated, offering clear advantages over other protocols.
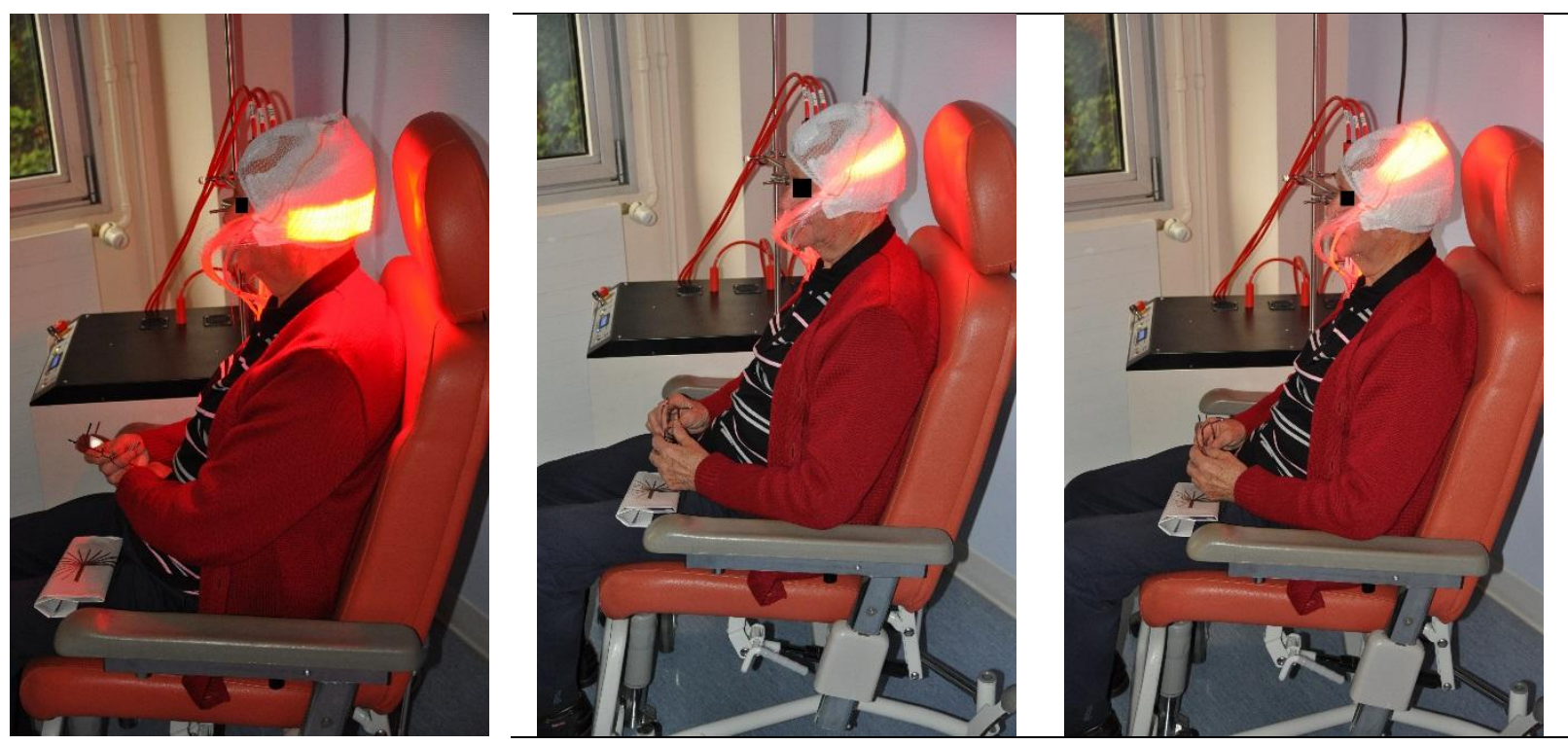

Figure 2: The three flexible light-emitting optical-fibre based fabrics involved in FLEXI-PDT sequentially emit $635 \mathrm{~nm}$ red light for one-minute resulting in a fractionated irradiation (1-minute light, 2 minutes' dark). 


\section{Phosistos protocol}

Developed within the Phosistos project funded by the European Commission (Project identifier: CIP-ICT-PSP-2013-7-621103) (http://www.phosistos.com/), P-PDT uses a 30-minute incubation with MAL cream under transparent occlusive dressing followed by $2.5 \mathrm{~h}$ of irradiation with a fabric-based biophotonic device without removing the dressing. As illustrated in Figure 1, this device consists of a power control unit including two $635 \mathrm{~nm}$ laser diodes with output power of $1 \mathrm{~W}$ each (Modulight, Tampere, Finland) and distributing red light at low-irradiance $\left(1.3 \mathrm{~mW} / \mathrm{cm}^{2}\right)$ to a light-emitting fabric that lines the inside of a cap (Texinov, Saint-Didier-de-la-Tour, France) (Figure 1). Made from bent optical fibres, this fabric is biocompatible, flexible and provides a homogeneous irradiation over a $21 \mathrm{~cm} \times 18 \mathrm{~cm}$ surface $\left(378 \mathrm{~cm}^{2}\right)$. Furthermore, an ergonomic helmet enables to keep the cap in place during the treatment. The device, classified as exempt risk group according to IEC 60601-2-57/2012, is configured to automatically start irradiation 30 minutes after it is turned on and stop it 2.5 hours later such that a total dose of approximately $12 \mathrm{~J} / \mathrm{cm}^{2}$ is delivered.
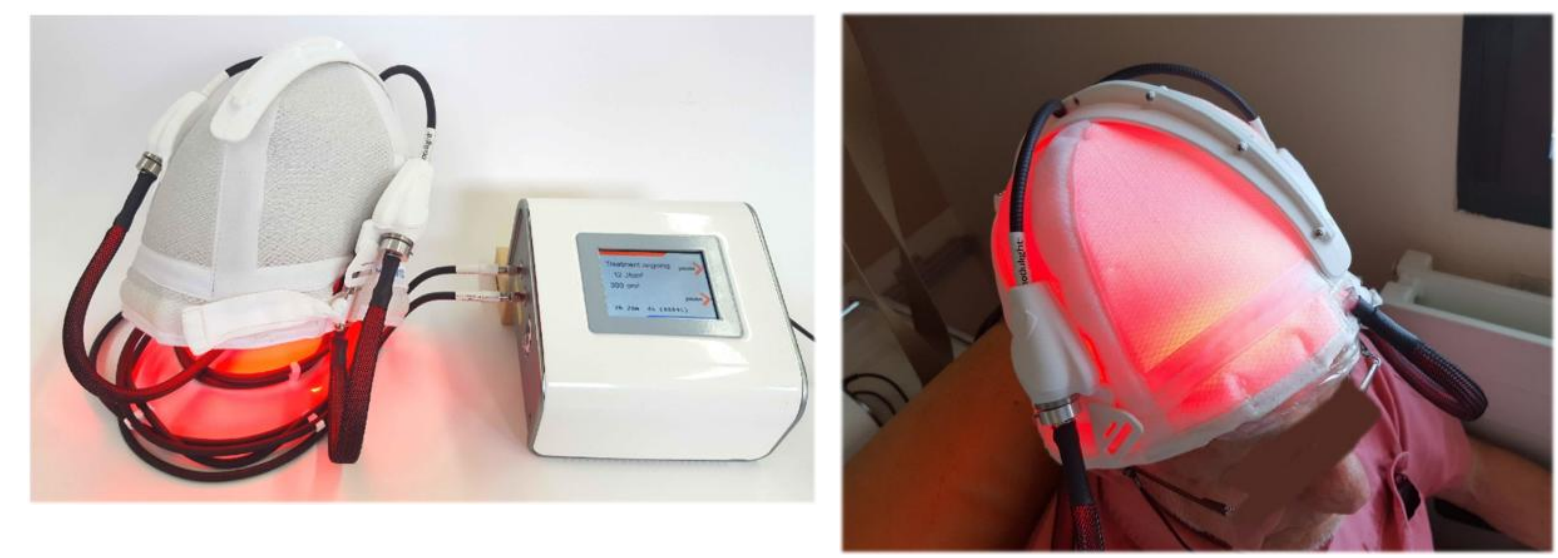

Figure 3: The fabric-based illumination device used for P-PDT: red light is emitted by an optical fibre-based fabric that lines the inside of a cap held in place by an ergonomic helmet.

\section{Treatment regimen}

The treatment regimen was similar for the 2 protocols. It includes one mandatory PDT session, and a second PDT session, identical to the first, in case of at least one remaining AK among the included ones at three months after the first PDT session. Follow-up visits were scheduled at 7 days, 3 months and 6 months after the first PDT session. If required, the second PDT session was performed within the three weeks following the 3-month follow-up visit.

At the screening visit, the patient's information, including demographic characteristics and Fitzpatrick skin type was recorded and a physical examination including dermatologic examination of the skin was performed. 
On the day of treatment, AK were counted, graded and divided into two areas with similar number and grade of AK. For accurate AK localization and follow-up, AK were traced on a transparent sheet placed over both the treatment areas. Randomization was performed by opening a sealed envelope specifying the protocol each area had to receive. Both the areas were prepared by removing crusts and gently scraping the lesion surface. Approximately 1 to $2 \mathrm{~g}$ of MAL cream (Metvixia, Galderma, France) was applied under transparent occlusive dressing (Tegaderm, 3M, London Ontario, Canada) in a $1 \mathrm{~mm}$ thick layer to the $\mathrm{AK}$ and surrounding normal skin (5-10 $\mathrm{mm}$ margin) of each area. Regarding the area randomized to receive $C$-PDT, an aluminium foil was placed over the transparent occlusive dressing. The cap involved in P-PDT was then immediately set up and fixed by the helmet and the power control unit was switched on so that irradiation required for P-PDT started thirty minutes later. After two hours and a half, P-PDT was completed. The cap, the helmet as well as all the dressings were removed and the MAL cream was washed off with saline solution. The area, which has just received P-PDT, was then protected with aluminium foil while the Aktilite CL128 (Galderma SA, Lausanne, Switzerland) was placed between 5 and $8 \mathrm{~cm}$ from the area randomized to receive C-PDT (illumination surface for the Aktilite CL128: $144 \mathrm{~cm}^{2}$ ) and programmed to deliver $37 \mathrm{~J} / \mathrm{cm}^{2}$ in seven to 10 minutes.

\section{RESULTS}

\section{Flexitheralight (FLEXI-PDT)}

\begin{tabular}{|c|c|c|c|}
\hline & FLEXI-PDT & C-PDT & $\begin{array}{c}\text { Superiority } p \text { value for } \\
\text { comparison between } \\
\text { randomised group }\end{array}$ \\
\hline $\begin{array}{c}\text { Number of lesions } \\
\text { Complete lesion response rate (\%) at 3 } \\
\text { months }\end{array}$ & 66.0 & 59.1 & $\mathrm{p}=0.070$ \\
\hline Complete lesion response rate (\%) at 6 & 84.0 & 76.8 & $\mathrm{p}=0.086$ \\
\hline Pain experienced during the $1^{\text {st }}$ treatment & $0.4 \pm 0.6$ & $5.0 \pm 2.6$ & $\mathrm{p}<0.0001$ \\
\hline Pain experienced during the $2^{\text {nd }}$ treatment & $0.2 \pm 0.5$ & $5.0 \pm 2.2$ & $\mathrm{p}<0.0001$ \\
\hline
\end{tabular}

Table 1: Complete response rate (lesion-level) achieved with FLEXI-PDT and C-PDT at 3 and 6 months [7].

27 patients were included in the study. Two patients dropped out for personal reasons before treatment. 25 patients with a total of 310 AK were treated and examined at three months after the treatment. Due to remaining AK, a second treatment session was required for 20 patients with a total of $252 \mathrm{AK}$. Between three and six months following the first treatment session, one patient dropped out due to a serious adverse event not related to the treatment and one patient did not return for the 6-month visit for personal reason. 23 patients with 286 AK therefore completed the study at 6 months.). Most of them had phototype II (76.0\%). A 
total of $156 \mathrm{AK}$, the majority of which were in grade I (42.3\%) and II (56.4\%), received FLEXIPDT. 154 AK (grade I: 42.2\%; grade II: 56.5\%; grade III: 1.3\%) received C-PDT.

At 3 month follow up, with 91 AK in complete response and 63 AK in incomplete response. CPDT achieved a lesion complete response rate at three months of $59.1 \%$ vs $66.0 \%$ with FLEXIPDT. At six months following treatment, the lesion complete response rate achieve $84.0 \%$ with FLEXI-PDT vs $76.8 \%$ with C-PDT. The response rate at six months for FLEXI-PDT (respectively, C-PDT) was around 1.3 (respectively, 1.3) times higher than that at three months. Similar local side effects, such as erythema and oedema, were observed with both FLEXI-PDT and C-PDT. Usual in dermatological PDT, these effects did not require any special care.

\section{Phosistos (P-PDT)}

Forty-seven patients were included in the study. One patient withdrew consent and did not receive treatment. Forty-six patients for a total of 560 AK were treated in a split-face manner with C-PDT (285 AK) and P-PDT (285 AK), and evaluated at 3 months of follow-up. Due to at least one remaining AK, 19 patients were required to undergo a second PDT session. Of these, one dropped out after the 3-month visit for fear of a pain as intense as that experienced with C-PDT during the first PDT session. As a result, only 18 patients (for a total of 105 remaining AK of the 204 initial AK at the first treatment session) were retreated. Forty-five patients completed the study at 6 months. All patients were men, aged $49-89$ years (mean age 72.4 years). Most patients had Fitzpatrick skin types II (63.8\%). Of the 285 AK randomized to receive C-PDT (respectively, P-PDT), 45.6\% (respectively, $44.9 \%$ ) were in grade I and $54.4 \%$ (respectively, 55.1\%) were in grade II.

At 3 month follow up, P-PDT was non-inferior to that obtained with C-PDT (79.3\% vs. 80.7\%, respectively. Six months following the first treatment session (after one PDT session for 27 patients and two PDT sessions for 18 patients). Whatever the protocol, almost all patients reported adverse effects throughout the study (100\% with C-PDT vs. $97.8 \%$ with P-PDT). However, the incidence of adverse effects was lower with P-PDT than with C-PDT (161 vs. 264).

The more important observation was the quasi-absence of pain with P-PDT. With all the pain scores ranging from 0 to 2.7, P-PDT was reported to be almost pain-free. Regarding the first PDT session (46 patients), the treatment-related pain at the end of irradiation is significantly lower with P-PDT compared to C-PDT (0.3 \pm 0.6 vs. $7.4 \pm 2.3, p<0.0001)$. The same finding was also observed for the second PDT session (18 patients) (Figure 4) $(0.2 \pm 0.4$ for P-PDT vs. $7.7 \pm$ 1.8, $\mathrm{p}<0.0001$ for C-PDT). 


\begin{tabular}{|c|c|c|c|}
\hline & P-PDT & C-PDT & $\begin{array}{c}\text { Superiority } p \text { value for } \\
\text { comparison between } \\
\text { randomised group }\end{array}$ \\
\hline Number of lesions & 280 & 280 & $\mathrm{p}=0.34$ \\
\hline $\begin{array}{c}\text { Complete lesion response rate (\%) at 3 } \\
\text { months }\end{array}$ & 80.7 & 79.3 & $\mathrm{p}=0.66$ \\
\hline Complete lesion response rate (\%) at 6 & 94.9 & 94.2 & $\mathrm{p}<0.0001$ \\
\hline Pain experienced during the $1^{\text {st }}$ treatment & $0.3 \pm 0.6$ & $7.4 \pm 2.3$ & $\mathrm{p}<0.0001$ \\
\hline Pain experienced during the $2^{\text {nd }}$ treatment & $0.2 \pm 0.4$ & $7.7 \pm 1.8$ & \\
\hline
\end{tabular}

Table 2: Complete response rate (lesion-level) achieved with P-PDT and C-PDT at 3 and 6 months [8].

\section{DISCUSSION}

Phosistos-PDT (P-PDT) is a revised version of the Flexitheralight protocol (Flexi-PDT). FlexiPDT, which consists of a 30-minute MAL incubation followed by a 2.5-hour fractionated irradiation with a light-emitting, fabric-based device emitting $635 \mathrm{~nm}$ red light at 12.3 $\mathrm{mW} / \mathrm{cm}^{2}$ irradiance and enabling homogeneous irradiation of the area to be treated, involves a $37 \mathrm{~J} / \mathrm{cm}^{2}$ light dose as C-PDT does. Based on a study that demonstrated the ability of two light sources with light doses lower than $15 \mathrm{~J} / \mathrm{cm}^{2}$ to completely photobleach PpIX [9] and on a study that reported successful PDT treatment of AK with red traffic lamps and a light dose of $3.5 \mathrm{~J} / \mathrm{cm}^{2}$ [10], the light dose was reduced from $37 \mathrm{~J} / \mathrm{cm}^{2}$ for Flexi-PDT to $12 \mathrm{~J} / \mathrm{cm}^{2}$ for P-PDT. Regarding the other irradiation parameters the irradiance (from $12.3 \mathrm{~mW} / \mathrm{cm}^{2}$ to 1.3 $\mathrm{mW} / \mathrm{cm}^{2}$ ) was also reduced and continuous irradiation was preferred to a fractionated one. The reduction in irradiance was motivated by several studies that have demonstrated similar PDT efficacy between variable irradiances $[11,12]$, while the use of fractionated irradiation, intended to primarily allow tissue reoxygenation, was discontinued due to the exclusive oxygen supply from the atmosphere of the skin up to a depth of $0.40 \mathrm{~mm}$ [13].

These changes in irradiation parameters have been successful since the non-inferiority in efficacy to C-PDT previously demonstrated with Flexi-PDT was also achieved with P-PDT.

\section{CONCLUSION}

With a 30-minute incubation with MAL followed by a $2.5 \mathrm{~h}$ irradiation with a light-emitting, fabric-based device, PDT can be conducted in all weather conditions, in any geographic location, year-round and benefits from the optimal adaptability of the flexible, light-emitting, fabrics to the treatment area. Most importantly, PDT with LEF of actinic keratosis has proved to be equally effective as Conventional PDT while being nearly pain-free. When compared to 
Daylight PDT, the main advantage of PDT with LEF is the possibility to perform the treatment in all weather conditions, in any geographic location, all year round. Consequently, PDT with LEF could therefore become the treatment of choice for AK.

\section{ACKNOWLEDGEMENTS}

The authors acknowledge the French National Research Agency (ANR) (Projet-ANR-12-EMMA0018) \& the European Commission for funding the Phosistos project under the Competitiveness and Innovation Framework Programme (CIP 621103). The authors thank the companies MDB TEXINOV for providing LEF and GALDERMA for providing Metvixia ${ }^{\circledR}$

\section{REFERENCES}

[1] Peris K, Fargnoli MC. Conventional treatment of actinic keratosis: an overview. Curr Probl Dermatol.; 46:108-14 (2015)

[2] Moseley H. Light distribution and calibration of commercial PDTLED arrays. Photochem Photobiol Sci ;4(11):911-4 (2005).

[3] Mordon S, Cochrane C, Tylcz JB, Betrouni N, Mortier L, Koncar V. Light emitting fabric technologies for photodynamic therapy. Photodiagnosis Photodyn Ther., Mar; 12(1):18 (2015).

[4] Cochrane C, Mordon SR, Lesage JC, Koncar V. New design of textile light diffusers for photodynamic therapy. Mater Sci Eng C Mater Biol Appl. Apr 1;33(3):1170-5 (2013).

[5] Thecua E. Vicentini C, Vignion-Dewalle A.S., Lecomte F., Deleporte, P., Mortier L., ; Szeimies R.M., Mordon S. , I. Light emitting fabric for photodynamic treatment of actinic keratosis. Proceedings Volume 10037, Photonics in Dermatology and Plastic Surgery; 100370L, SPIE,. San Francisco (2017).

[6] Tylcz, J.B., C. Vicentini, and S. Mordon, 4 - Light emitting textiles for a photodynamic therapy, in Smart Textiles and their Applications, V. Koncar, Editor., Woodhead Publishing: Oxford. p. 71-87 (2016).

[7] Vicentini C, Vignion-Dewalle AS, Thecua E, Lecomte F, Maire C, Deleporte P, Béhal H, Kerob D, Duhamel A, Mordon S, Mortier L. Photodynamic therapy for actinic keratosis of the forehead and scalp: a randomized, controlled, phase II clinical study evaluating the noninferiority of a new protocol involving irradiation with a light-emitting, fabricbased device (the Flexitheralight protocol) compared with the conventional protocol involving irradiation with the Aktilite CL 128 lamp. Br J Dermatol., Apr;180(4):765-773 (2019). 
[8] Mordon S, Vignion-Dewalle AS, Abi-Rached H, Thecua E, Lecomte F, Vicentini C, Deleporte P, Béhal H, Kerob D, Hommel T, Duhamel A, Szeimies RM, Mortier L. The conventional protocol versus a protocol including illumination with a fabric-based biophotonic device (the Phosistos protocol) in photodynamic therapy for actinic keratosis: a randomized, controlled, non-inferiority clinical study. Br J Dermatol., Apr 25 (2019)

[9] Lerche CM, Heerfordt IM, Heydenreich J et al. Alternatives to Outdoor Daylight Illumination for Photodynamic Therapy--Use of Greenhouses and Artificial Light Sources. Int J Mol Sci ; 17: 309. (2016)

[10] Enk CD, Levi A. Low-irradiance red LED traffic lamps as light source in PDT for actinic keratoses. Photodermatol Photoimmunol Photomed; 28: 332-4 (2012).

[11] Apalla Z, Sotiriou E, Panagiotidou D et al. The impact of different fluence rates on pain and clinical outcome in patients with actinic keratoses treated with photodynamic therapy. Photodermatol Photoimmunol Photomed; 27: 181-5. (2011)

[12]Langmack K, Mehta R, Twyman P et al. Topical photodynamic therapy at low fluence rates--theory and practice. J Photochem Photobiol B; 60: 37-43 (2001).

[13] Stucker M, Struk A, Altmeyer P et al. The cutaneous uptake of atmospheric oxygen contributes significantly to the oxygen supply of human dermis and epidermis. I Physiol; 538: 985-94 (2002) 